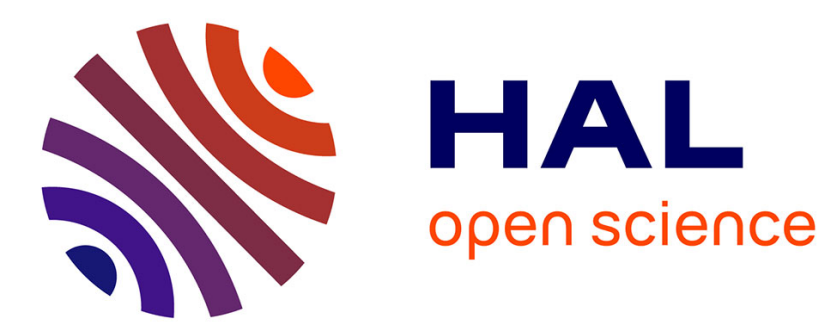

\title{
Analysis of in-Cylinder Pressure Oscillation and its Effect on Wall Heat Transfer
}

Mateos Kassa, Thomas Leroy, Anthony Robert, Fabien Vidal- Naquet

\section{To cite this version:}

Mateos Kassa, Thomas Leroy, Anthony Robert, Fabien Vidal- Naquet. Analysis of in-Cylinder Pressure Oscillation and its Effect on Wall Heat Transfer. International Journal of Engine Research, 2021, 22 (7), pp.2189-2202. 10.1177/1468087420932364 . hal-03352688

\section{HAL Id: hal-03352688 \\ https://hal-ifp.archives-ouvertes.fr/hal-03352688}

Submitted on 23 Sep 2021

HAL is a multi-disciplinary open access archive for the deposit and dissemination of scientific research documents, whether they are published or not. The documents may come from teaching and research institutions in France or abroad, or from public or private research centers.
L'archive ouverte pluridisciplinaire HAL, est destinée au dépôt et à la diffusion de documents scientifiques de niveau recherche, publiés ou non, émanant des établissements d'enseignement et de recherche français ou étrangers, des laboratoires publics ou privés. 


\title{
Analysis of in-cylinder pressure oscillation and its effect on wall heat transfer
}

\author{
Mateos Kassa, Thomas Leroy, Anthony Robert, and Fabien Vidal- \\ Naquet
}

IFP Energies nouvelles

\begin{abstract}
In-cylinder pressure oscillations in internal combustion engines have been associated with increased heat losses and damages to the engine components. The links between the acoustic waves and the increased heat transfer (and potentially ensuing engine damages) have not yet been well understood. In this study, a high fidelity Large Eddy Simulation (LES) model incorporating an auto-ignition model is used to simulate the combustion process and the associated pressure oscillation at various engine operating conditions. The study serves to develop a better understanding of the acoustic waves in a combustion chamber and their effect on wall heat transfer. First, a simplified model of the pressure oscillations is proposed and shown to accurately characterize the pressure in the combustion chamber. Second, the simplified pressure model and acoustic theory are leveraged to develop a model of the incylinder gas velocities. Last, a heat transfer model is presented that takes into consideration the pressure/velocity oscillations and the inherent acoustic properties of the trapped gas. The increase in heat transfer is shown to primarily stems from an increased heat transfer coefficient due to the velocity oscillations of the trapped gas. The results are consistent with previously observed experimental measurements of the heat flux in the presence of pressure oscillations.
\end{abstract}

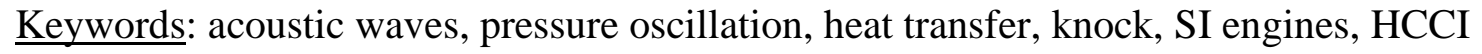

\section{Introduction}

The ever-growing energy and environmental concerns have led to strict regulations on vehicle fuel consumption and emissions in many countries around the world. Out of the more than one billion vehicles in the world, more than $60 \%$ of them are equipped with gasoline Spark Ignition (SI) engines [1]. These engines are considered to have significant potential for further improvement in engine efficiency and are projected to continue to play an important role in the transportation industry for a very long time [1]. Several engine technologies such as direct injection, turbocharging, pre-chamber combustion, and advanced combustion strategies including Homogeneous Charge Compression Ignition (HCCI) and Spark Assisted Compression Ignition (SACI) are leveraged to improve the thermal efficiency and reduce the emissions of the engines [2]. These modifications generally feature rapid and short combustions, which helps to reduce the thermal losses. One inadvertent consequence, however, is the resulting pressure gradients and pressure oscillations in the combustion chamber due to the rapid heat release rates. These pressure oscillations are often a result of engine knock, but are also common in combustion modes featuring rapid combustion rates. 
The pressure oscillations have been shown to lead to increased wall heat transfer resulting in fuel efficiency losses and, in some cases, to damages of the engine components $[3,4,5]$. This study seeks to develop a better understanding of the pressure oscillation and its effect on the in-cylinder gases and the heat transfer to the engine walls.

The most common instances of pressure oscillations in the combustion chamber arise due to engine knock. The auto-ignition of pockets of air-fuel mixture in an SI engine, commonly known as engine knock, is one of the main obstacles preventing today's engines from attaining optimal high load operations and subsequently higher thermal efficiencies. The instances of engine knock and the emanating pressure oscillations have been directly associated to engine failures, primarily destructions of the engine piston that are indicative of increased heat transfer. The knock damage mechanisms have been extensively studied over the years. Nates et al. [6] mention the need for a knock intensity measurement based on a damage related threshold. Their study indicates that a principal aspect of knocking combustion that initiates damage is the elevated global heat flux. Kowada et al. [7] also acknowledge the importance of heat transfer towards the knock induced damage. To that end, they develop a new knock indicator that indirectly evaluates the heat transfer by accounting for the frequency of knocking cycles, the volumetric efficiency, and the engine speed. Cavina et al. [8] emphasize that temperature strongly influences instantaneous material properties and a primary source of engine failure is the degradation of the piston's mechanical strength due to elevated surface temperatures. Their study concludes that a precise estimation of the extratransferred heat due to knock is essential for a meaningful evaluation of the knock induced risks. These studies collectively argue that the additional heat transfer to the wall (primarily to the piston) in knocking instances stems from the pressure oscillations and is a primary factor contributing to the engine damage.

In addition to knock related concerns in traditional SI engines, the increased heat transfer to the engine walls is persistent in advanced combustion modes such as HCCI where significant pressure oscillations are present. Several experimental studies [9, 10, 11, 4] have sought to quantify and model the wall heat transfer in the presence of pressure oscillations. Chang et. al. [9] used fast response thermocouples embedded in the piston and cylinder head surface to measure instantaneous wall temperature and heat flux for an HCCI engine. Their study suggests an improved model for the heat transfer by modifying the fitting coefficients of the heat transfer model initially proposed by Woschni [12]. Similar studies are conducted by Hensel et al. [10]. Broekaert et al. [11] have conducted an assessment of the models developed by both Chang et. al. [9] and Hensel et al. [10], as well as prior models developed for traditional SI and compression ignition (CI) engines [12, 13, 14]. The study concludes that none of the models were able to accurately capture the effects of different engine settings on the heat flux; furthermore, the study highlights that the models lack some parameters that have a significant influence on the heat transfer during HCCI combustion (in the presence of pressure oscillations).

To answer to this lack of model representativeness, some studies [3, 15] have leveraged numerical tools to evaluate the pressure oscillations' impact on the heat transfer to the cylinder walls. Winkler [3] set out to find the physical mechanisms responsible for the increase in heat transfer to the in-cylinder walls when pressure oscillations are present. In his work, rapid heat release rates were simulated using LES in order to analyze the effect of the resulting pressure oscillations. The study concludes that the mechanism found for the enhanced heat transfer is the rapid transport of high-temperature fluid from the heat source towards the wall by the velocity fluctuations emerging from the pressure wave. Similarly, 
Corti et. al. [15] leverage a Reynolds Averaged Navier-Stokes (RANS) simulation to quantify the wall heat transfer in knocking conditions and propose a modified Woschni heat transfer model. The proposed model still resulted in an underestimation of the heat flux in highly knocking conditions, and reflects the lack of an accurate evaluation of the pressure oscillations' impact on heat transfer.

The contribution of this paper consists in providing a better understanding of the effects of pressure oscillations on the heat transfer to the cylinder walls. To that end, a high-fidelity Large Eddy Simulation (LES) integrating auto-ignition modeling is used to compute incylinder conditions at various spark advance conditions of a particular engine operating point [16]. In this work, the acoustic effects emanating from the auto-ignition of the fuel and reflected as pressure and velocity oscillations are analyzed. Three results are presented. First, a simplified model of the pressure oscillations is proposed and shown to accurately characterize the pressure in the combustion chamber. Second, the simplified pressure model and acoustic theory are leveraged to develop a model of the in-cylinder gas velocities. Last, a heat transfer model is presented that takes into consideration the pressure/velocity oscillations and the inherent acoustic properties of the trapped gas.

The paper is organized as follows. In the next section, a description of the numerical tools constituting the LES is given along with a description of the engine used for the study. Following that, a brief discussion highlighting the preliminary observations of the work is presented and is followed by a detailed discussion of the results of the study. Final remarks regarding the utilization of the results are presented with concluding remarks at the end.

\section{Numerical Setup: Large Eddy Simulation (LES)}

The LES calculations taken as an input for this study are performed using the AVBP code [17, 18] which solves the multi-species, compressible, reactive Navier-Stokes equations on unstructured and moving meshes. More details on this LES database are available in [16]. The engine used for this study is a 1.2L turbocharged direct injection engine with a compression ratio of 10.3. Further details about the engine specification can be found in Table 1. The simulation consists of an operating condition at 5500 RPM and full load. Fifteen consecutive cycles were simulated (without knock) to generate a variation of aerodynamic conditions. These cycles were used to prepare the initial conditions of the simulation and determining the turbulent flow characteristics at the beginning of compression and ignition. The turbulence characteristics include volume-averaged turbulence kinetic energy, the distributions of local velocities, temperatures, and fuel concentrations. Subsequently, a variation of spark timing was implemented to yield various levels of pressure oscillations associated to knock. All the initial conditions of spatially varying velocities, temperatures, and fuel concentrations were kept the same though the knock takes place in every cycle with different levels of pressure oscillations which was induced by a variation of spark timing. A total of 23 cycles were used for the analysis presented in this study.

Table 1. Engine characteristics and simulated operating point.

\begin{tabular}{ll}
\hline Engine capacity (swept volume) & $1.2 \mathrm{~L}$ \\
Compression ratio & 10.3 \\
Rotational speed & $5500 \mathrm{rev} / \mathrm{min}$ \\
IMEP & $19 \mathrm{bar}$ \\
\hline
\end{tabular}


Fuel

Spark timing sweep
SP95-E10

From 27.5 CAD before the reference spark timing to the reference spark timing

For this work, time advancement is explicit and convection is discretized using a second order (in space and time) centered finite volume Lax-Wendroff scheme [19]. Furthermore, the subgrid scale turbulence is modeled by a Smagorinsky model [20] with a constant set to $\mathrm{Cs}=0.18$. The direct injection requires taking into account spray for which the Lagrangian formalism [21] is used to simulate the liquid phase. In order to accurately incorporate the effects of abnormal (knocking) combustion, the ignition by the spark plug, the premixed flame propagation and the auto-ignition processes are collectively modeled. The spark ignition is modeled with the ISSIM-LES model [22], whereas the flame front propagation is described using the ECFMLES premixed combustion model [23, 24]. The tabulated autoignition model (TKI-LES) $[25,26]$ is used to model the reaction rate linked to the occurrence of auto-ignition in the fresh gases. Based on local conditions, an auto-ignition reaction rate is extracted from a look-up table for each node of the mesh, and used in the species transport equations. This look-up table is built from complex chemistry simulations using a TRF surrogate with $42.8 \%$ isooctane, $13.7 \%$ n-heptane, $43.5 \%$ toluene and the LLNL kinetic mechanism considering 1388 species and 5935 reactions [27]. An important aspect of these models is that auto-ignition and premixed flame are described by two independent progress variables which allow the analysis of the contribution of each phenomenon during a knocking cycle.

Due to the complexity introduced by the moving parts, a total of seventy tetrahedral meshes are needed to simulate a full cycle, each constituting between 1.7 and 9.2 Million cells. The mean cell size is approximately $0.05 \mathrm{~mm}$ at the residual lift for intake and exhaust valves. Cell size in the chamber is around $0.7 \mathrm{~mm}$ during the main part of the cycle and $0.5 \mathrm{~mm}$ during combustion phases. Finally, the mesh size is refined down to $0.2 \mathrm{~mm}$ in the vicinity of the spark plug during ignition.

With regards to the boundary conditions, temporal signals resulting from a GT-Power calculation are imposed as inlet and outlet boundary conditions for each LES cycle. Wall temperatures, which are a first order parameter to study knock, are estimated using a conjugate heat transfer (CHT) based on RANS calculations. Visualizations of wall temperatures, as well as additional information regarding the numerical method, simulation conditions (boundary conditions, initial and operating conditions), pictorial view of the engine, and validation of the model can be found in [16].

\section{Preliminary observations}

As previous studies [11, 15] have already shown, conventional heat transfer models significantly misrepresent the wall heat transfer in the presence of pressure oscillations. This observation is further confirmed through the simulation results presented in this study. Figure 1 illustrates the normalized heat transfer to the piston for an engine cycle (through LES calculations) for the same operating condition with and without pressure oscillations (specified as knocking and nominal respectively). Figure 1 displays both the local heat transfer (left) at a location specified by the Cartesian coordinates from the center of the cylinder and the surface averaged heat transfer (right). The heat transfer rate calculation is independent to the vertical position (chosen here as $\mathrm{z}=0 \mathrm{~mm}$ - piston position at TDC) as the 
pressure oscillation is invariant along the z-axis; this will be further discussed in the next section. The nominal case for each knocking cycle was derived using a low pass $(3 \mathrm{kHz})$, noncausal filter of the in-cylinder pressure, temperature, and gas velocity. A sample cycle is used to illustrate the effect of knock on the wall heat transfer.
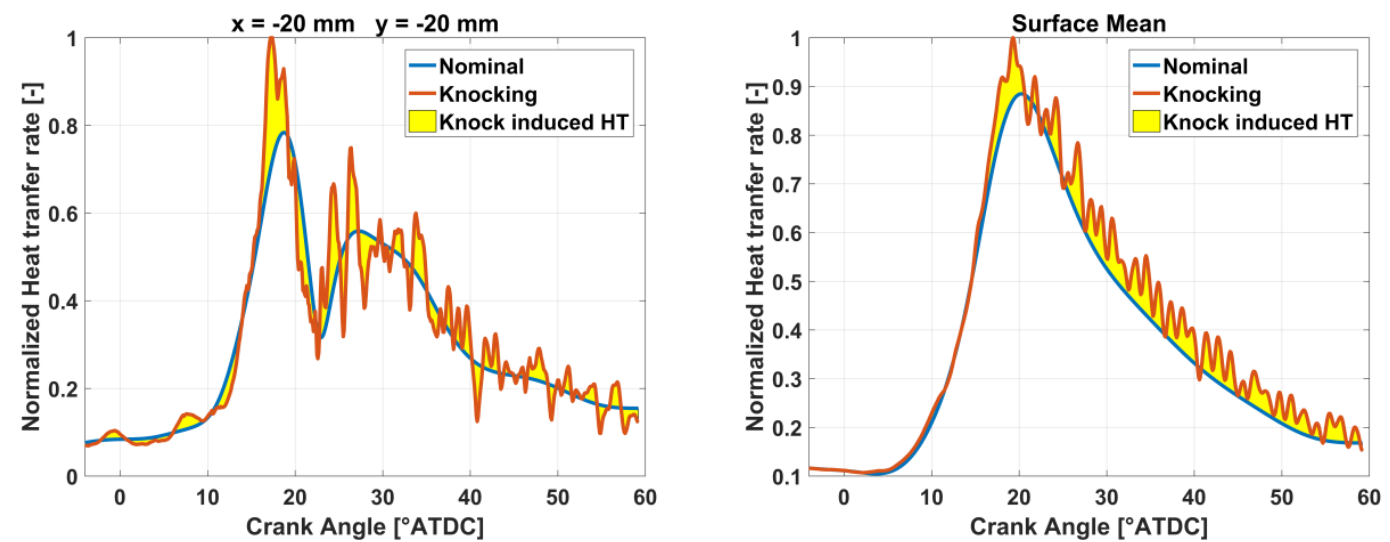

Figure 1. Illustration of local and average wall heat transfer with and without the presence of pressure oscillations (knock).

The results illustrated in Figure 1 suggest an additional heat transfer due to engine knock for the particular cycle (yellow area relative to area under blue curve on right plot). An interesting observation from Figure 1 is that although the local heat transfer displays an oscillation of the knocking heat transfer curve around the nominal heat transfer curve, the surface averaged heat transfer curves show that the knocking case is consistently above the nominal case. The oscillating pressure can locally lead to more or less heat transfer compared to the nominal case as the pressure during knocking instances can locally be higher or lower than the nominal pressure. However, when the surface averaged heat transfer is analyzed, the additional heat transfer are bound to cancel out for the most part. Furthermore, there will also be additional heat transfer due to velocity oscillations; unlike the pressure oscillations, the velocity oscillations consistently lead to increased gas speeds during knocking instances compared to the nominal case. As such the surface mean heat transfer, as reflected in Figure 1, is consistently higher in knocking cases compared to the nominal case. This will be further discussed in the following sections.This observation is consistent throughout the rest of the cycles studied and illustrates the additional knock (or pressure oscillations)-induced heat transfer.

Similar observations were also reported in experimental studies by Dejima et al [28] and Lu et. al. [5]. These observations further suggest the influence of the pressure oscillations and encourage its consideration for more accurate heat transfer estimations. A series of results in the representation of the in-cylinder pressure oscillation, its impact on the gas velocities, and the resulting effects on the wall heat transfer will be discussed in the subsequent sections.

\section{Results and discussions}

In this section, the acoustic waves present in the combustion chamber and characterized by the pressure and velocity oscillations are analyzed for a better understanding of their effect on 
the wall heat transfer. The following subsections will discuss the steps bridging the pressure oscillations and the additional wall heat transfer. First, it is shown that the resolution of the wave equation in the combustion chamber permits a simplified representation of the incylinder pressure oscillations. Second, it is shown that with the use of the principles of conservation of momentum and the simplified representation of the pressure oscillations, an accurate model of the gas velocities can be developed. Lastly, an alternative heat transfer model is proposed that takes into consideration the acoustic effects in the combustion chamber.

\section{Simplified model of in-cylinder pressure oscillations}

Unlike experimental engine cycles where the pressure measurement is generally limited to a single point, the LES based cycles allow a holistic perception of the in-cylinder pressure oscillations throughout the combustion chamber. The pressure at each point in the combustion chamber permits a thorough investigation of its effects. In this section, a simplified model of the pressure oscillation (via the resolution of the wave equation) is shown to adequately characterize the pressure in the combustion chamber as observed in the LES results.

The wave equation, as described in Eq. 1 for pressure, is a second-order linear partial differential equation that can be used to describe acoustic waves under specific conditions:

$$
\Delta P=\frac{1}{c^{2}} \frac{\partial^{2} P}{\partial t^{2}}
$$

Eq. 1

where $P$ is the pressure, $c$ is the speed of sound, and $t$ represents time.

Specifically, the hypotheses necessary for its applicability in accurately describing the pressure oscillations in a combustion chamber are as follows [29, 30]:

- The fluid is bi-variant, meaning it can be fully described by two thermodynamic variables

- The fluid has no viscosity or thermal conductivity

- There are no chemical reactions, no heat transfer, and no mass transfer

- There are no volume forces

It should be noted that none of these statements are generally true, yet their assumption serves as a tool to formulate a simplified representation of the pressure oscillations in the chamber with minimal error. Indeed, as initially presented by Draper in 1935 [31, 32], the wave equation can be leveraged to describe the oscillations of the pressure (acoustic waves) in the combustion via a modal decomposition. The resolution of the wave equation via separation of variables and the assumption of a perfect cylinder geometry yields the following representation of the pressure throughout the combustion chamber:

$$
P(r, \theta, z, t)=\sum_{s, b, g} A_{s, b, g} \cdot J_{s}\left(\beta_{s, b} \cdot r\right) \cdot{ }_{\cos }^{\sin }(s \theta) \cdot \cos \left(\frac{g \pi}{h} z\right) \cdot \cos (2 \pi n t)
$$




$$
n=c \cdot \sqrt{\frac{\beta^{2}}{4 \pi^{2}}+\frac{g^{2}}{4 h^{2}}}
$$

where $P(r, \theta, z, t)$ is the pressure in the combustion chamber at a location specified by the cylindrical coordinates $r, \theta$, and $z$ at a time $t ; J_{s}\left(\beta_{s, b} \cdot r\right)$ is Bessel's function of the first kind and $\mathrm{s}^{\text {th }}$ order evaluated at $\beta_{s, b} \cdot r$, where $\beta_{s, b}$ are constants satisfying the condition that the product of $\beta_{s, b}$ and the cylinder radius is the $b^{\text {th }}$ root of the derivative of Bessel's function (first kind, $\mathrm{s}^{\text {th }}$ order); the notation ${ }_{\cos }^{\sin }(s \theta)$ is used to represent a linear sum of the sine and cosine components; $g$ is a whole number; $h$ is the height of the cylinder; $A_{s, b, g}$ are constants depending upon the amplitude of the vibration and the particular modes involved; $n$ is the oscillation frequency of the particular modes; and $c$ is the speed of sound.

Once again, the consideration that the combustion chamber is a perfect cylinder is generally untrue due to the shape of the cylinder head as well as that of the piston. Despite this approximation and the assumed hypotheses for the applicability of the wave equation, the findings of this study will show that the modal decomposition illustrated in Eq. 2 can still be leveraged for a sufficiently accurate representation of the in-cylinder pressure.

As will be discussed later in this section, the modal decomposition, in addition to providing a concise representation of the in-cylinder pressure, also serves as an effective tool for calculating the resulting gas velocity oscillations. In the first steps prior to exploiting the modal decomposition of the in-cylinder pressure, the LES cycles are used to evaluate the applicability and limitations of the model. Although the representation of the pressure in Eq. 2 stems from a direct solution to the wave equation, the previously mentioned hypotheses and approximations can compromise the applicability of the model.

In order to evaluate the modal decomposition, a 2-dimensional slice of the in-cylinder pressure is fitted to the pressure modes throughout the combustion process. The spatial shape of the modes is given by the expression $\mathrm{J}_{\mathrm{s}}\left(\beta_{\mathrm{s}, \mathrm{b}} \cdot \mathrm{r}\right) \cdot{ }_{\cos }^{\sin }(\mathrm{s} \theta)$ and is illustrated in Figure 2 for various values of $s$ and $b$. The frequency of each mode as a function of the speed of sound are also indicated in Figure 2 above each image. The speed of sound is on the order of $1000 \mathrm{~m} / \mathrm{s}$ suggesting a frequency around $7.8 \mathrm{kHz}$ for the mode $\mathrm{s}=1, \mathrm{~b}=1$. 

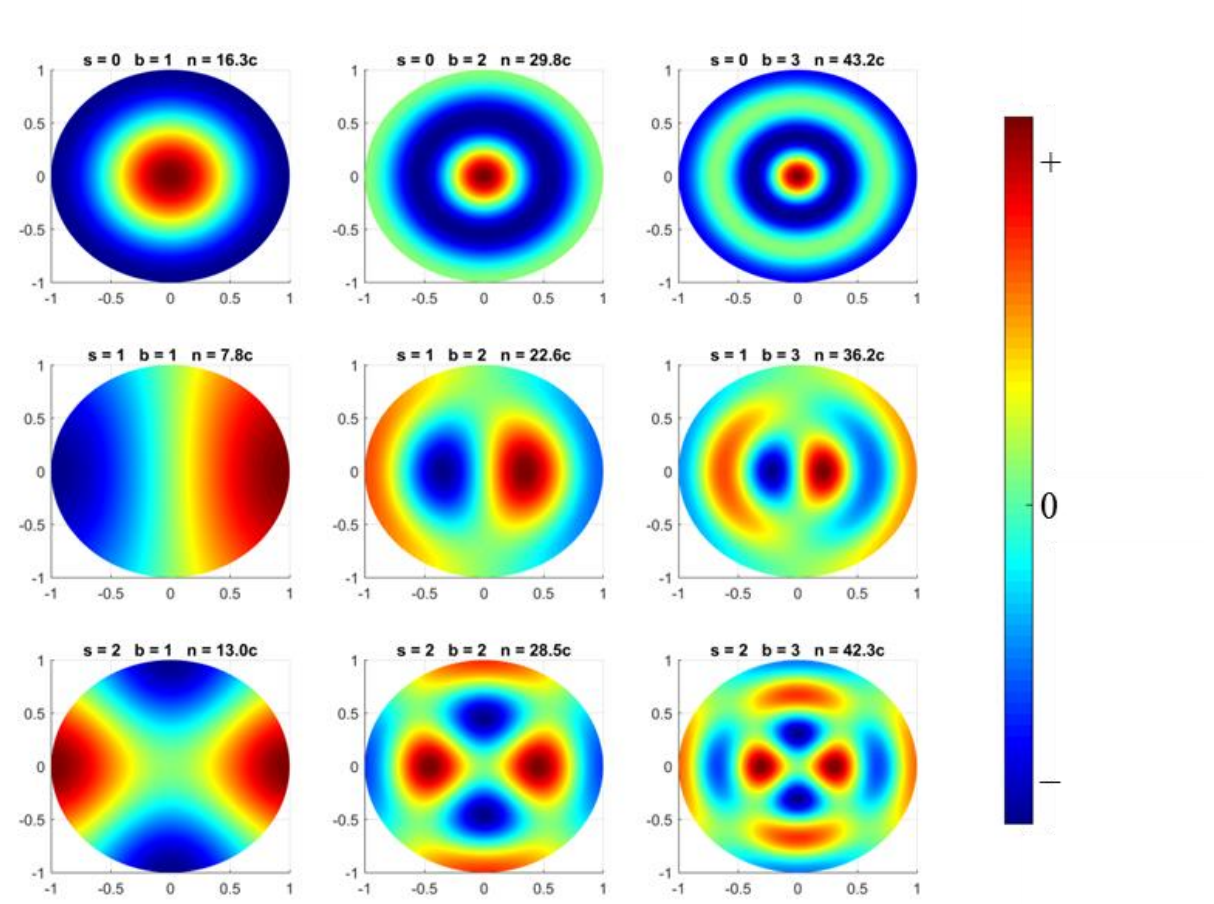

Figure 2. Illustration of a selected number of modes representing the spatial distribution of the pressure

It should be noted that only the $\cos (s \theta)$ counterparts of each pair of modes are displayed. Also, since the analysis relies on an axial slice, the expression $\cos \left(\frac{g \pi}{h} z\right)$ is ignored, yielding purely two-dimensional pressure oscillation modes. The consideration of an axial slice is, however, not limiting in the analysis of the pressure throughout the combustion chamber. In fact, the observation from the LES results, as well as previous studies [33, 34], suggest that the modes with $g \neq 0$ exhibit very high frequencies (can be seen in Eq. 2) and have negligible presence relative to the other modes. Thus, the exclusive use of modes with $g=0$ is reasonable approach.

The fitting process consists of equating the two-dimensional in-cylinder pressure to a sum of the modes. The fitting is done at each time step between $10^{\circ}$ before top dead center (BTDC) and $60^{\circ}$ after top dead center (ATDC) with a resolution of $0.1^{\circ}$. The fitting parameters are calculated at each time step using a linear regression. A total of 7950 data points are used to characterize the pressure trace at each instant of time. The fitting process relied on 55 pairs of modes with s ranging from 0 to 10 and considering the first 5 Bessel roots for each value of $\mathrm{s}$, as shown in Eq. 3. This results in total of 105 modes (note: $s=0$ has no $\sin (s \theta)$ component) with an additional pseudo-mode that is simply a constant throughout the plane capturing the spatial mean of the pressure. The pressure fitting process, thus, consists of a dimension reduction from 7950 data points to 106 parameters in fully describing the in-cylinder pressure:

$$
\begin{aligned}
& P(r, \theta, t)=P_{0}(t)+\sum_{s=0}^{10} \sum_{b=1}^{5} A_{s, b}^{\cos } \cdot \cos (2 \pi n t) \cdot J_{s}\left(\beta_{s, b} \cdot r\right) \cdot \cos (s \theta) \quad \text { Eq. } 3 \\
& +\sum_{s=1}^{10} \sum_{b=0}^{5} A_{s, b}^{\sin } \cdot \cos (2 \pi n t) \cdot J_{s}\left(\beta_{s, b} \cdot r\right) \cdot \sin (s \theta)
\end{aligned}
$$


where $P_{0}(t)$ is the spatial mean of the pressure at time $t$; the fitted parameters are $A_{s, b}^{c o s}$. $\cos (2 \pi n t)$ and $A_{s, b}^{\sin } \cdot \cos (2 \pi n t)$ corresponding to the modes $J_{s}\left(\beta_{s, b} \cdot r\right) \cdot \cos (s \theta)$ and $J_{s}\left(\beta_{s, b} \cdot r\right) \cdot \sin (s \theta)$ respectively for a sweep of $s$ and $b\left(n=c \frac{\beta_{s, b}}{2 \pi}\right)$.

The fitting result shows good agreement between the LES results and the model. First, the fitting is accurate with less than 0.2 bar error $\left(95^{\text {th }}\right.$ percentile) across the plane all throughout the combustion process. The fitting accuracy is illustrated in Figure 3 which superposes the fitted pressure trace onto the LES derived results at four locations in the combustion chamber. Both the raw pressure trace and the filtered pressure (above $3 \mathrm{kHz}$ ) are simultaneously plotted. Separate y-axes are used to illustrate the filtered and non-filtered components. Similar results are obtained at other locations throughout the combustion chamber, and the fitting yields just as accurate results for all the cycles studied.
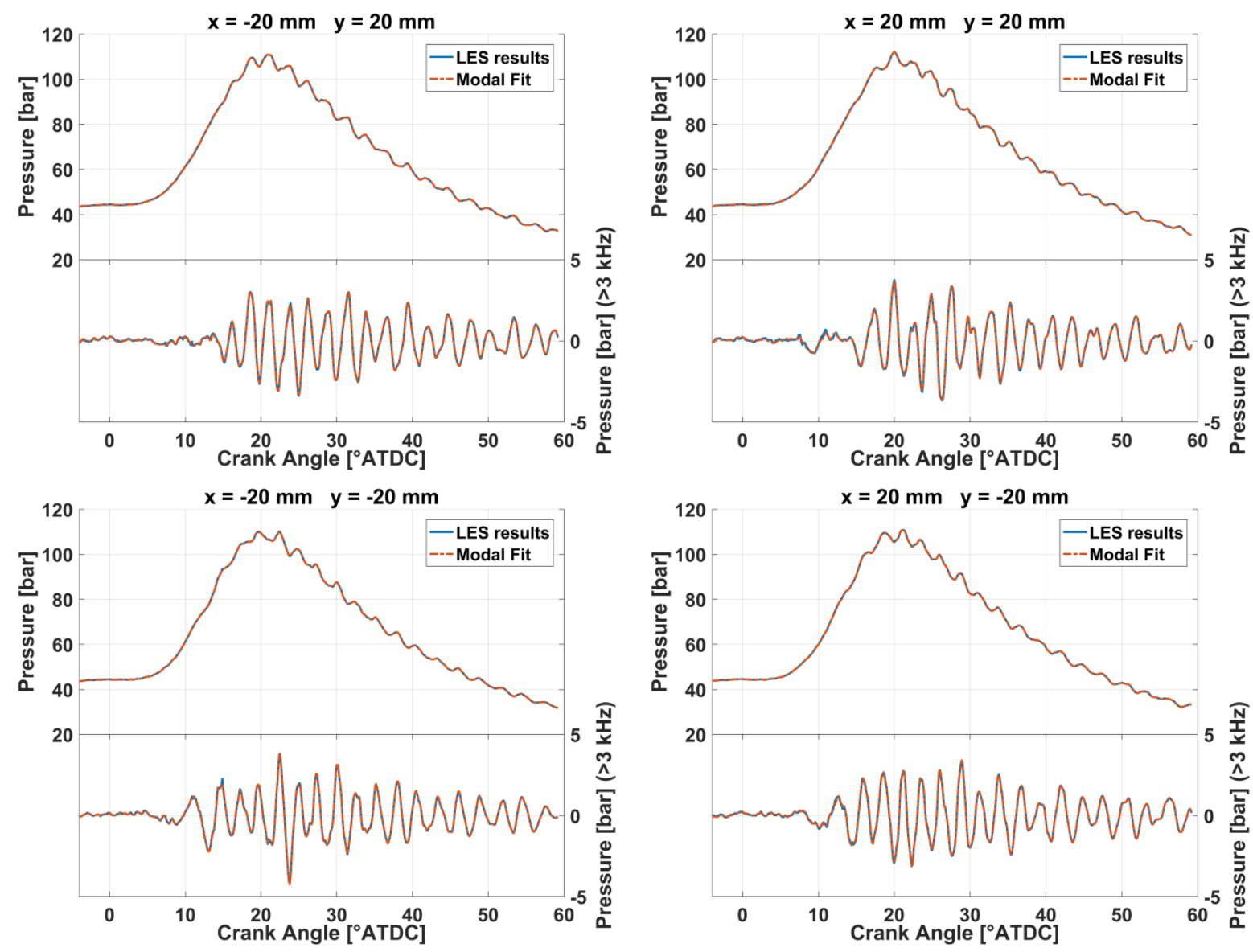

Figure 3. Illustrating of pressure modal fitting ( $>3 \mathrm{kHz}$ component simultaneously plotted)

Second, and more importantly, the fitting parameters are coherent in time (note: the fitting is done at each crank angle with a resolution of $0.1^{\circ}$ ) and are periodic as suggested by the theory. The fitting results for a selected number of modes are illustrated in Figure 4. Similar results are obtained for the remaining modes, which supports the applicability of a modal representation to characterize the in-cylinder pressure. 

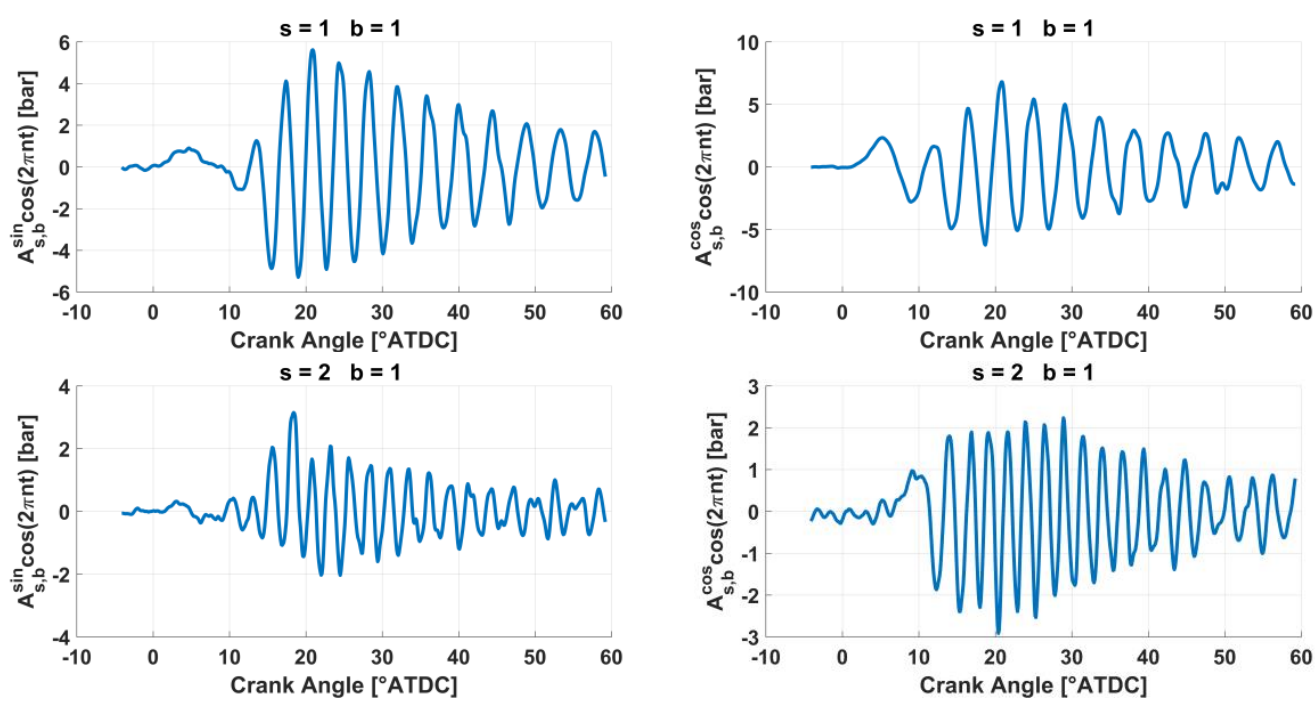

Figure 4. Sample illustration of few modes and resulting fitting amplitude (sine and cosine components)

Figure 4 illustrates the fitting results for the pairs of the two most prevalent modes $(s=1, b=1$ and $s=2, b=1$ ). Besides the continuity in time and the periodicity illustrated in Figure 4, further observations affirm the underlying physical properties of the fitting process. The piston motion, which represents work done by the fluid (in contradiction to the wave equation hypotheses), leads to a decaying amplitudes of the fitting parameters. Furthermore, the irregularity of the cylinder geometry due to a pent-roof cylinder head results in a non-uniform frequency evolution in time; as such, the frequency of the oscillations are higher when the piston is near top-dead center (due to a smaller radius).

Lastly, the frequency of the oscillations observed in the fitted expression of each mode was analyzed to examine the coherence with the modal frequencies imposed by the wave equation. The power spectral density (PSD) of each oscillation is computed to identify the dominant frequency of the oscillations. As can be seen in Figure 5, the frequency resulting from the modal fitting is well correlated with the theoretical expectation $\left(n=c \frac{\beta_{s, b}}{2 \pi}\right)$. Some outliers, highlighted with the red circles, can be observed in Figure 5; these points are identified to stem from numerical errors as their amplitude is very small and both the fitting and PSD analysis succumb to interferences from other modes. 

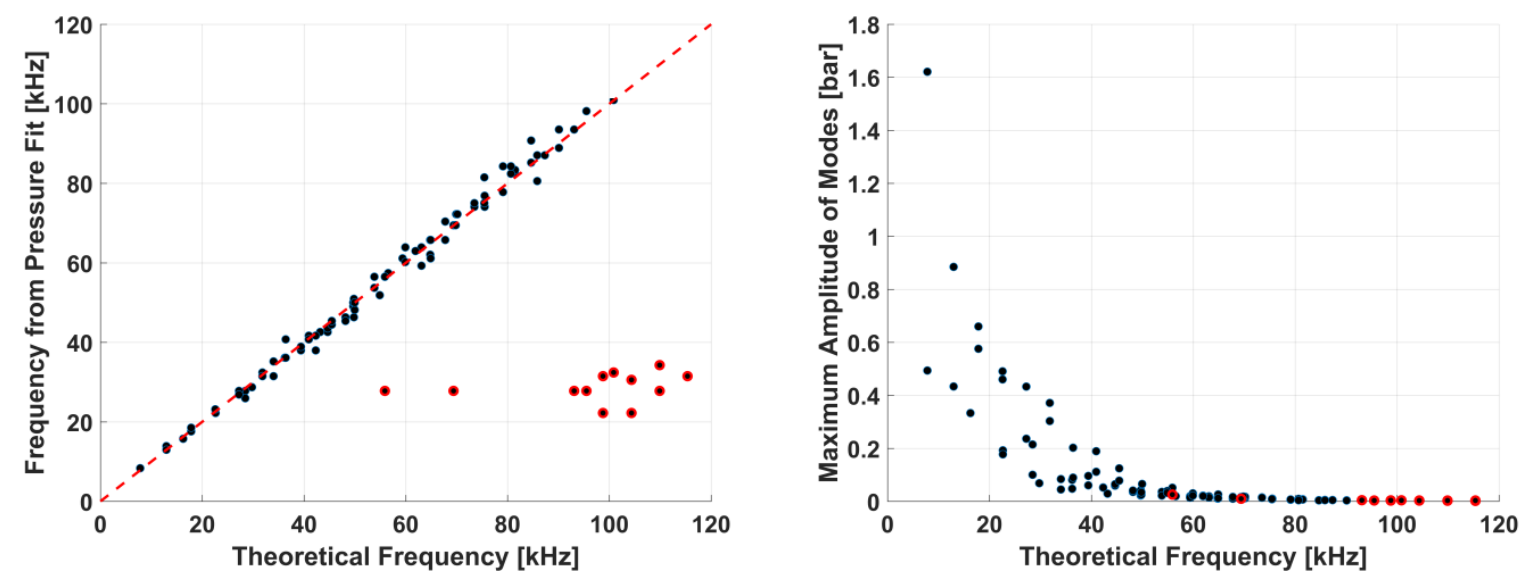

Figure 5. Comparison of modal frequency derived from fitting with theoretical expectation (outliers in red)

Figure 5 also illustrates the importance of each mode via the illustration of the maximum amplitudes of each mode. At each resonant frequency, the amplitudes of a pair of modes (the $\sin (\mathrm{s} \theta)$ and $\cos (\mathrm{s} \theta)$ counterparts) are displayed. As can be observed, the mode with a frequency around $8 \mathrm{kHz}$ is the most important one (which is commonly known experimentally and leveraged for knock detection), followed by another one around $12 \mathrm{kHz}$; nevertheless, this study further illustrate that other modes with higher frequencies also have non-negligible importance. It is evident from Figure 5 that there is a global trend in which higher frequency modes are less and less important. This was observed consistently throughout the study of all 23 cycles, and has also been previously proposed in experimental studies [35, 30].

The representation of the in-cylinder pressure via the modal decomposition shown in Eq. 3 was repeated for a total of 23 LES cycles. In each of the cycles, similar results were obtained validating the applicability of the modal decomposition to accurately characterize the pressure oscillations. The next section discusses how the modal characterization can be utilized to calculate in-cylinder velocity oscillations, which play an important role in the heat transfer coefficient.

\section{In-cylinder velocity model based on modal representation of in- cylinder pressure}

As briefly mentioned previously, the representation of the in-cylinder pressure via its modal components provides an effective tool for a characterization of the in-cylinder gas velocities. The oscillation of the velocities (especially at the surface of the cylinder walls) plays an important role on the heat transfer coefficient, and thus merits accurate evaluation. The incylinder velocity can be represented as the sum of its main components:

$$
\vec{u}=\overrightarrow{u_{0}}+\overrightarrow{u_{1}}+\vec{\varepsilon}
$$

where $\vec{u}$ represents the total velocity, $\overrightarrow{u_{0}}$ represents the nominal velocity dictated by the aerodynamics, $\overrightarrow{u_{1}}$ represents the velocity oscillation dictated by acoustic effects, and $\vec{\varepsilon}$ is used to represent other potential velocity components such as turbulence. 
Additionally, the principles of conservation of momentum yield the following equation, which will be used to derive the in-cylinder gas velocity oscillations:

$$
\rho_{0} \frac{\partial \overrightarrow{u_{1}}}{\partial t}+\nabla P_{1}=0
$$

where $\rho_{0}$ is the mean density in the combustion chamber, $u_{1}$ is the velocity oscillations, and $P_{1}$ is the pressure oscillations.

A simultaneous gradient (spatial) and integration (temporal) of the in-cylinder pressure is required to derive the in-cylinder gas velocity oscillations. The modal representation of the incylinder pressure facilitates this process since the spatial and temporal terms are separately defined. Thus, the in-cylinder gas velocity oscillations can be directly calculated from the incylinder pressure trace. The resulting expression for the in-cylinder velocity oscillations (derived from Eq. 3 and Eq. 5) is as follows:

$$
\overrightarrow{u_{1}}(r, \theta, t)=\sum_{s, b}\left(\nabla\left(J_{s}\left(\beta_{s, b} \cdot r\right) \cdot \cos _{\cos }^{\sin }(s \theta)\right) \cdot \int_{t_{0}}^{t_{0}+\Delta t} \frac{A_{s, b} \cdot \cos (2 \pi n t)}{\rho_{0}} d t\right)
$$

Eq. 6

It should be noted that the gradient of the pressure modes yields two vectorial components, which can be expressed in cylindrical form as follows:

- for pressure modes in the form of $J_{s}\left(\beta_{s, b} \cdot r\right) \cdot \cos (s \theta)$,

$\left\{\begin{array}{l}u_{1, r}(r, \theta, t)=\sum_{s, b}\left(\left(-\beta_{s, b} \cdot J_{s+1}\left(\beta_{s, b} \cdot r\right) \cdot \cos (s \theta)-\frac{s \cdot J_{s}\left(\beta_{s, b} \cdot r\right)}{r}\right) \cdot \int_{t_{0}}^{t_{0}+\Delta t} \frac{A_{s, b}^{c o s} \cdot \cos (2 \pi n t)}{\rho_{0}} d t\right) \\ u_{1, \theta}(r, \theta, t)=\sum_{s, b}\left(-s \cdot J_{s}\left(\beta_{s, b} \cdot r\right) \cdot \sin (s \theta) \cdot \int_{t_{0}}^{t_{0}+\Delta t} \frac{A_{s, b}^{c o s} \cdot \cos (2 \pi n t)}{\rho_{0}} d t\right)\end{array}\right.$

- for pressure modes in the form $J_{s}\left(\beta_{s, b} \cdot r\right) \cdot \sin (s \theta)$,

$$
\left\{\begin{array}{l}
u_{1, r}(r, \theta, t)=\sum_{s, b}\left(\left(-\beta_{s, b} \cdot J_{s+1}\left(\beta_{s, b} \cdot r\right) \cdot \sin (s \theta)-\frac{s \cdot J_{s}\left(\beta_{s, b} \cdot r\right)}{r}\right) \cdot \int_{t_{0}}^{t_{0}+\Delta t} \frac{A_{s, b}^{s i n} \cdot \cos (2 \pi n t)}{\rho_{0}} d t\right) \\
u_{1, \theta}(r, \theta, t)=\sum_{s, b}\left(s \cdot J_{s}\left(\beta_{s, b} \cdot r\right) \cdot \cos (s \theta) \cdot \int_{t_{0}}^{t_{0}+\Delta t} \frac{A_{s, b}^{s i n} \cdot \cos (2 \pi n t)}{\rho_{0}} d t\right)
\end{array}\right.
$$

Eq. 8

The integration is computed throughout the combustion period where the velocity calculation is desired. Furthermore, the axial uniformity of the pressure oscillations $(g=0)$ dictates the absence of axial velocity oscillations $\left(\overrightarrow{u_{1, z}}=0\right)$. Figure 6 provides a visual demonstration that illustrates the calculation of the velocity oscillations through a treatment of the in-cylinder pressure modal representation. The gradient of the spatial components of the pressure modes are used to derive those of the velocity. Furthermore, the integration of the pressure mode's amplitudes divided by the density yields the amplitude for the velocity modes. These steps are applied to each of the 105 modes and summed together to generate the in-cylinder velocity oscillations. 


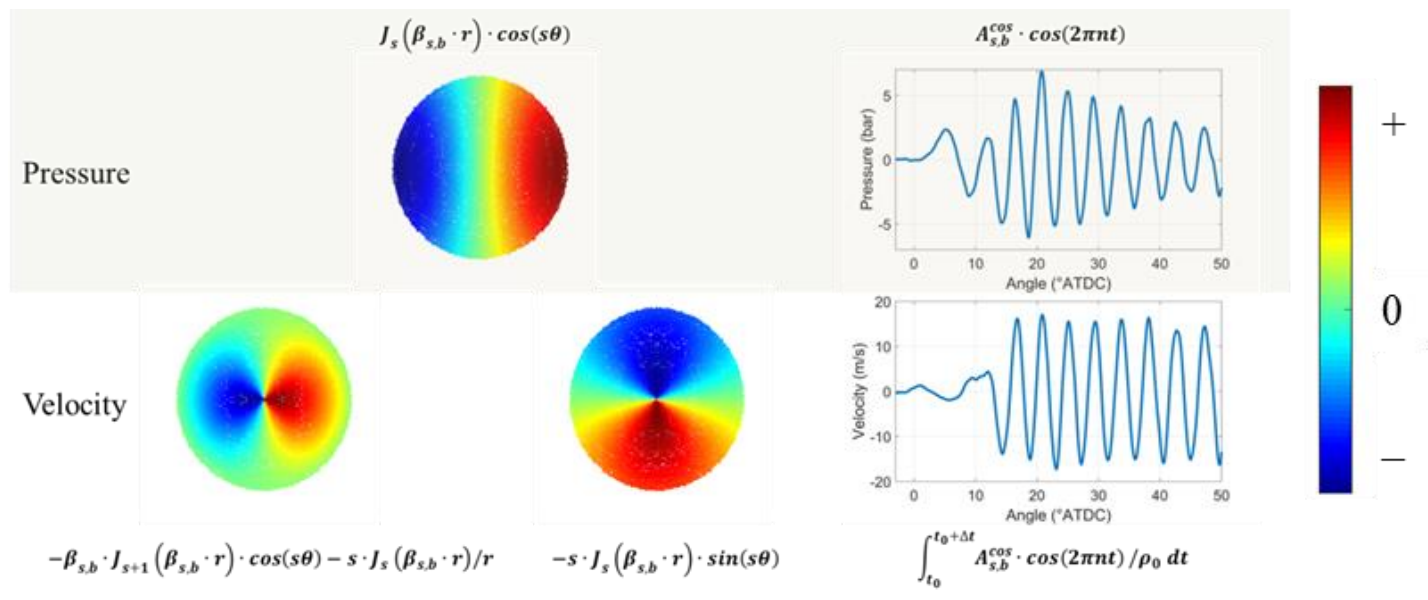

Figure 6. Illustration of calculation of in-cylinder velocity oscillations from modal representation of in-cylinder pressure

Figure 7 compares the in-cylinder gas velocities derived from the modal pressure with the actual velocities calculated in the LES computation. Once again, four locations evenly spaced out across the axial plane are used to evaluate the velocity oscillations. Both the total velocity $\left(u_{r}\right)$ as well as the velocity oscillations $\left(u_{1, r}\right)$ are depicted in Figure 7 . The radial component is used in the illustration, but similar results are obtained for the circumferential component.
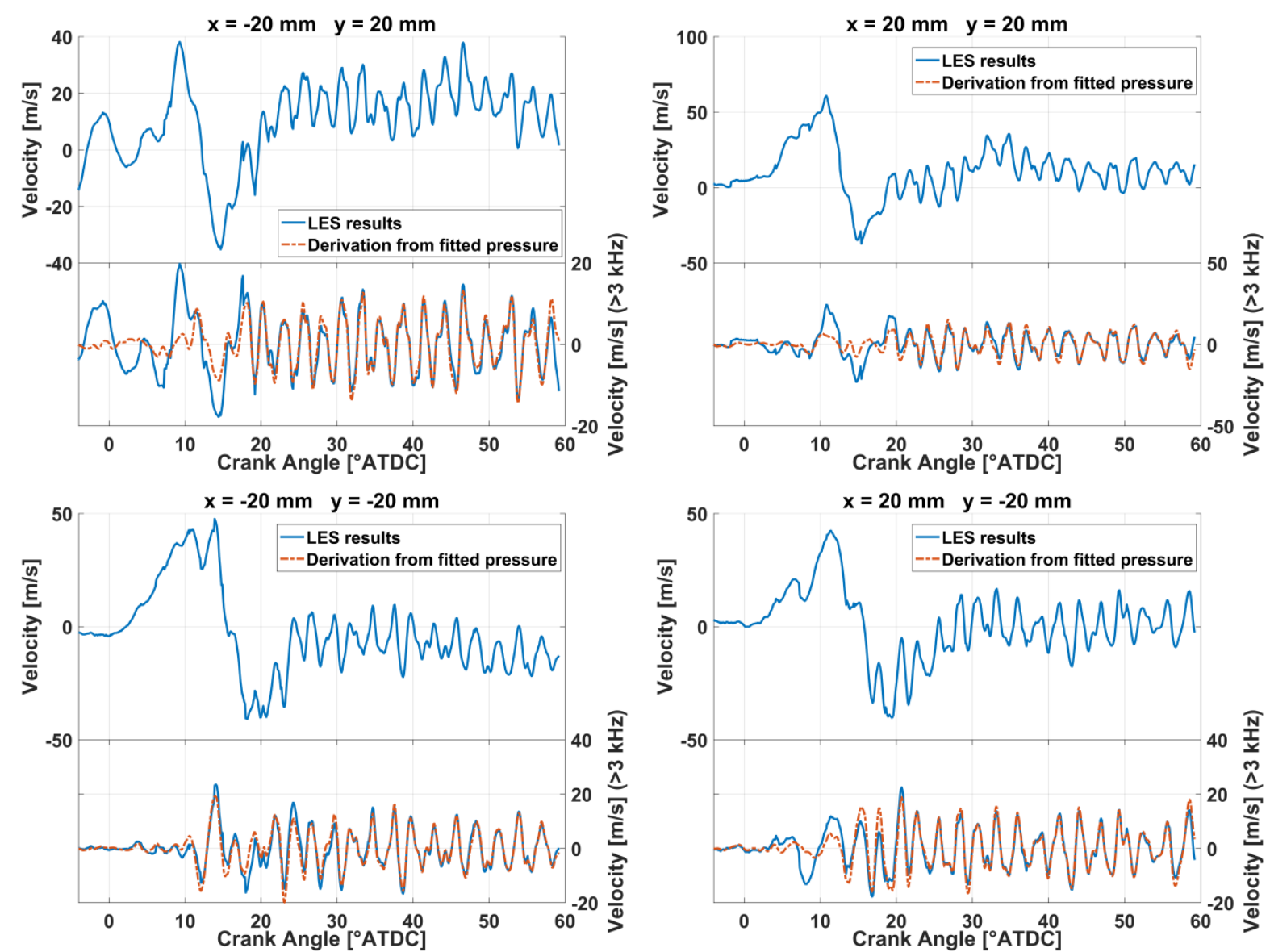

Figure 7. Comparison of modal pressure derived in-cylinder velocity oscillations with actual LES results 
As can be observed in Figure 7, the velocities derived from the fitted pressure trace match very well with the LES results. Some deviations can be observed in the initial stages of the combustion (prior to $20^{\circ}$ ATDC); these suggest that the assumed hypotheses (specifically assuming the absence of chemical reactions and heat transfer) introduce a non-negligible source of error. Additional errors stemming from turbulence effects are also expected to affect the results. Nevertheless, the trends of the velocity oscillations both spatially and temporally are generally well captured by the model in the subsequent stages of the combustion. Figure 7 also provides an idea of the magnitude of the velocity oscillations (relative to the total velocity) present in these instances alluding to the important role these oscillations can have on the heat transfer coefficient.

It should be noted that the discussions in this section and the illustration of Figure 7 is focused on the oscillations of the in-cylinder gas velocities (as opposed to the nominal velocity). The nominal velocity, generally represented as $\overrightarrow{u_{0}}$, is not a function of the pressure oscillations, but rather primarily affected by the piston speed and other aerodynamic effects such as swirl and tumble motions. The observations of Figure 7 are consistent across all the 23 cycles that were studied. The in-cylinder velocity oscillations are accurately evaluated from a modal representation of the in-cylinder pressure. The magnitude of the in-cylinder velocity oscillations are generally significant $(\sim 10-20 \mathrm{~m} / \mathrm{s})$ and persistent throughout the combustion process, suggesting the importance of its consideration for heat transfer coefficient calculations.

\section{Heat transfer model in presence of pressure oscillations}

The last result consists of incorporating the in-cylinder gas velocities into the calculation of the heat transfer coefficient for a better heat transfer model. The convective heat transfer model acknowledges the importance of the gas velocity near the surface in computing the heat transfer coefficient; nevertheless, conventional heat transfer models fail to take into consideration the additional gas velocity in the presence of pressure oscillations.

In this section, the surface of the piston is chosen to illustrate the heat transfer calculation. The heat loss to the wall is calculated using a Newtonian model:

$$
Q=\iint_{S} h \cdot\left(T-T_{w}\right) d A
$$

where $Q$ is the heat transfer, $h$ is the heat transfer coefficient, $T$ is temperature of the cylinder gas, $T_{w}$ is the cylinder wall temperature, $d A$ represents an incremental area, and the integral is over a surface $S$ (ex: piston head).

The heat transfer coefficient is calculated via a correlation between Nusselt, Reynolds, and Prandt numbers:

$$
\left(\frac{h L}{k}\right)=C \cdot\left(\frac{\rho u L}{\mu}\right)^{m} \cdot\left(\frac{c_{p} \mu}{k}\right)^{n}
$$


where $h$ is the heat transfer coefficient, $L$ is a characteristic length, $k$ is the thermal conductivity, $\rho$ is the density, $u$ is a characteristic speed, $\mu$ is the dynamic viscosity, $c_{p}$ is the heat capacity; and, $C, m$, and $n$ are fitting coefficients.

For the application of engine wall heat transfer, previous studies [13, 14] and most notably the works by Woschni [12] have derived the following expression for the heat transfer coefficient (written here in its general form) with various propositions for the fitting parameters:

$$
h=\kappa \cdot P^{\alpha} \cdot T^{\beta} \cdot u^{\gamma}
$$

where $P$ is the pressure, $T$ is the temperature, $u$ is the gas characteristic speed; and $\kappa, \alpha, \beta$, and $\gamma$ are fitting constants.

The heat transfer coefficient is shown to be predominantly a function of the in-cylinder pressure, temperature, and gas velocities. The analysis in the prior sections highlights the resulting effects of the acoustic phenomenon on the pressure and velocity oscillations, which are generally neglected in the heat transfer calculation.

The proposed heat transfer coefficient is thus represented as follows to take into consideration the acoustic effects:

$$
h=\kappa \cdot\left(P_{0}+P_{1}\right)^{\alpha} \cdot\left(T_{0}+T_{1}\right)^{\beta} \cdot\left\|\overrightarrow{u_{0}}+\overrightarrow{u_{1}}\right\|^{\gamma}
$$

where $P_{0}, T_{0}$, and $\overrightarrow{u_{0}}$ are the gas pressure, temperature, and velocity respectively in the absence of pressure oscillations; and, $P_{1}, T_{1}$, and $\overrightarrow{u_{1}}$ are used to represent the acoustic wave contributions.

In addition to the pressure and velocity oscillations, prior studies [3] have suggested that the pressure waves will affect the temperature field through compression and expansion of the gas. The temperature oscillations were estimated from a linearized ideal gas law as follows:

$$
T_{1}=\frac{P_{1}}{\rho_{1} \cdot c_{p}}
$$

where $c_{p}$ is the specific heat capacity at constant pressure.

These temperature oscillations were not observed in the LES results and are considered negligible in this study. Furthermore, the consideration that $P_{1} \ll P_{0}$ encourages a simplification of the heat transfer coefficient calculation via a first order Taylor approximation:

$$
\left(P_{0}+P_{1}\right)^{\alpha} \approx P_{0}^{\alpha}+\alpha \cdot P_{1} \cdot P_{0}^{\alpha-1}
$$

which yields

$$
h \approx \kappa \cdot P_{0}{ }^{\alpha} \cdot T_{0}{ }^{\beta} \cdot\left\|\overrightarrow{u_{0}}+\overrightarrow{u_{1}}\right\|^{\gamma}+\kappa \cdot \alpha \cdot P_{1} \cdot P_{0}{ }^{\alpha-1} \cdot T_{0}{ }^{\beta} \cdot\left\|\overrightarrow{u_{0}}+\overrightarrow{u_{1}}\right\|^{\gamma}
$$


where even the second term is negligible relative to the first. The ratio between the two terms $\left(\frac{\alpha P_{1}}{P_{0}}\right)$ suggests a relative importance of the second term which is less than $4 \%$ (with a consideration of $\alpha=0.8$ and $\left.\frac{P_{1}}{P_{0}}<0.05\right)$.

The resulting expression illustrates that the pressure oscillation term $\left(P_{1}\right)$ has little effect in the calculation of the heat transfer coefficient; however, the effects of the acoustic waves are bound to be reflected in the heat transfer coefficient calculation via the velocity expression.

Note,

$$
\left\|\overrightarrow{u_{0}}+\overrightarrow{u_{1}}\right\|^{\gamma}=\left(\sqrt{\left(u_{0, r}+u_{1, r}\right)^{2}+\left(u_{0, \theta}+u_{1, \theta}\right)^{2}}\right)^{\gamma}
$$

where $u_{0, r}$ and $u_{0, \theta}$ represent the radial and circumferential gas velocities respectively in the absence of acoustic waves; and, $u_{1, r}$ and $u_{1, \theta}$ represent the acoustic contributions (as derived in Eq. 7-10).

It should be noted that the expression $\left\|\overrightarrow{u_{0}}+\overrightarrow{u_{1}}\right\|$ only considers the speed across the plane (ignoring axial velocity stemming primarily from the piston motion). At the piston surface, where the heat transfer calculation is desired, the axial velocity is zero. The gas speed parallel to the piston plane is, thus, the pertinent parameter required for the heat transfer coefficient calculation.

Unlike the pressure oscillation term, the velocity oscillation $\overrightarrow{u_{1}}\left(u_{1, r}\right.$ and $\left.u_{1, \theta}\right)$ plays an important role in the heat transfer coefficient due to the importance of the constituting terms relative to the nominal velocity terms. Figure 8 illustrates the impact of the acoustic waves of the gas speeds locally across the piston plane. It can be seen that the effect of the acoustic waves on the gas speeds are consistently present throughout the combustion process (at least up to $60^{\circ} \mathrm{ATDC}$ ). Unlike the gas speeds under nominal conditions (w/o acoustic waves), the gas speeds in the presence of the acoustic waves display high frequency $(\sim 8-40 \mathrm{kHz})$ oscillations. This behavior of the gas speeds is observable throughout the combustion chamber, and the specific fluctuations at 4 distinct locations across the piston plane can be seen in Figure 8. 

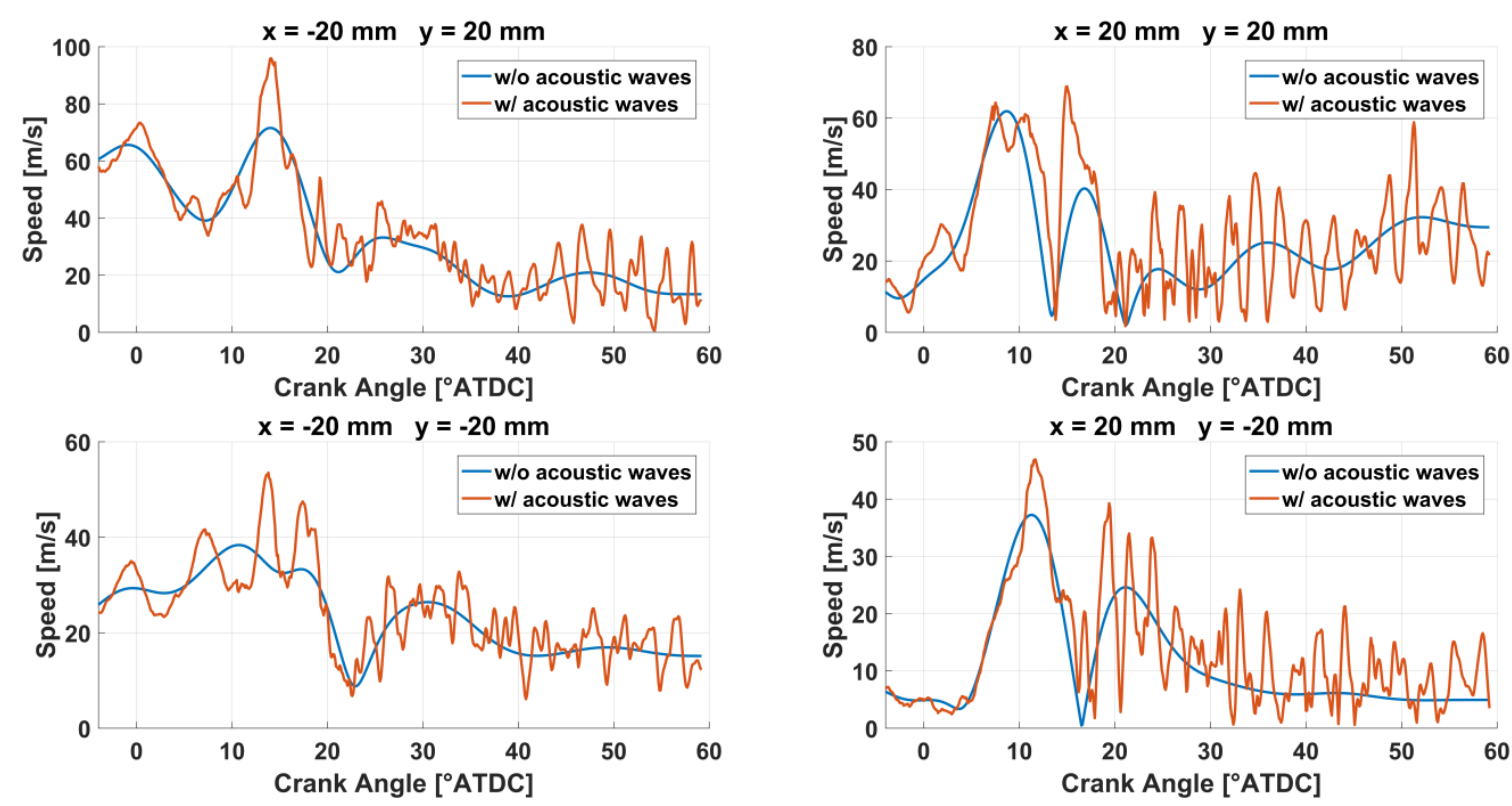

Figure 8. In-cylinder gas velocity near piston surface with and without acoustic waves

In addition to the local speeds of the gas in the combustion chamber, the illustration of the average gas speed across the plane provides an additional perspective into the effects of the acoustic waves. The average speed across the plane is representative of the average heat transfer coefficient as $P_{0}$ and $T_{0}$ are expected to be relatively constant across the plane. Figure 9 provides an illustration of the plane-averaged gas speed.

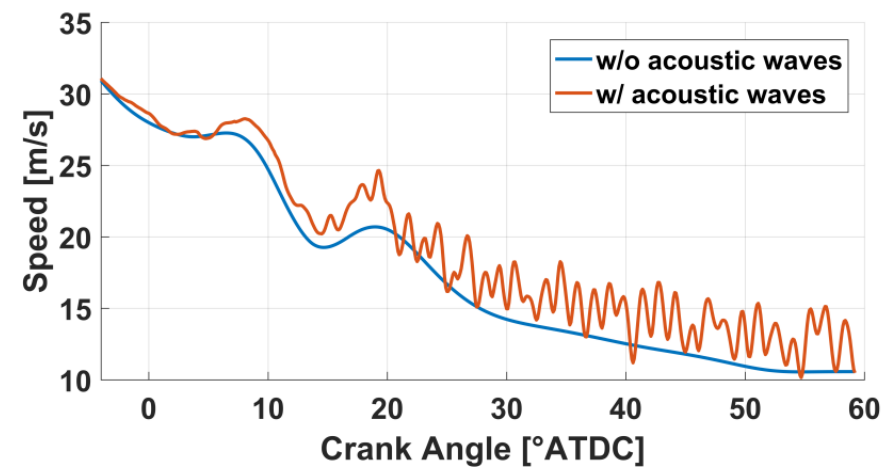

Figure 9. In-cylinder mean gas velocity near piston surface with and without acoustic waves $\left(\overline{|u|}=\frac{\int|u| d A}{\int d A}\right)$.

As can be seen in Figure 9, the presence of acoustic waves leads to consistently higher gas motion on the piston surface. The net effect of the speed oscillations observed in Figure 8 (averaged over the complete piston surface) is depicted as a global increase of the average gas speed. This effect is consistently observed throughout all the LES cycles studied, and is presumed to stem from the shape of the velocity modes. 

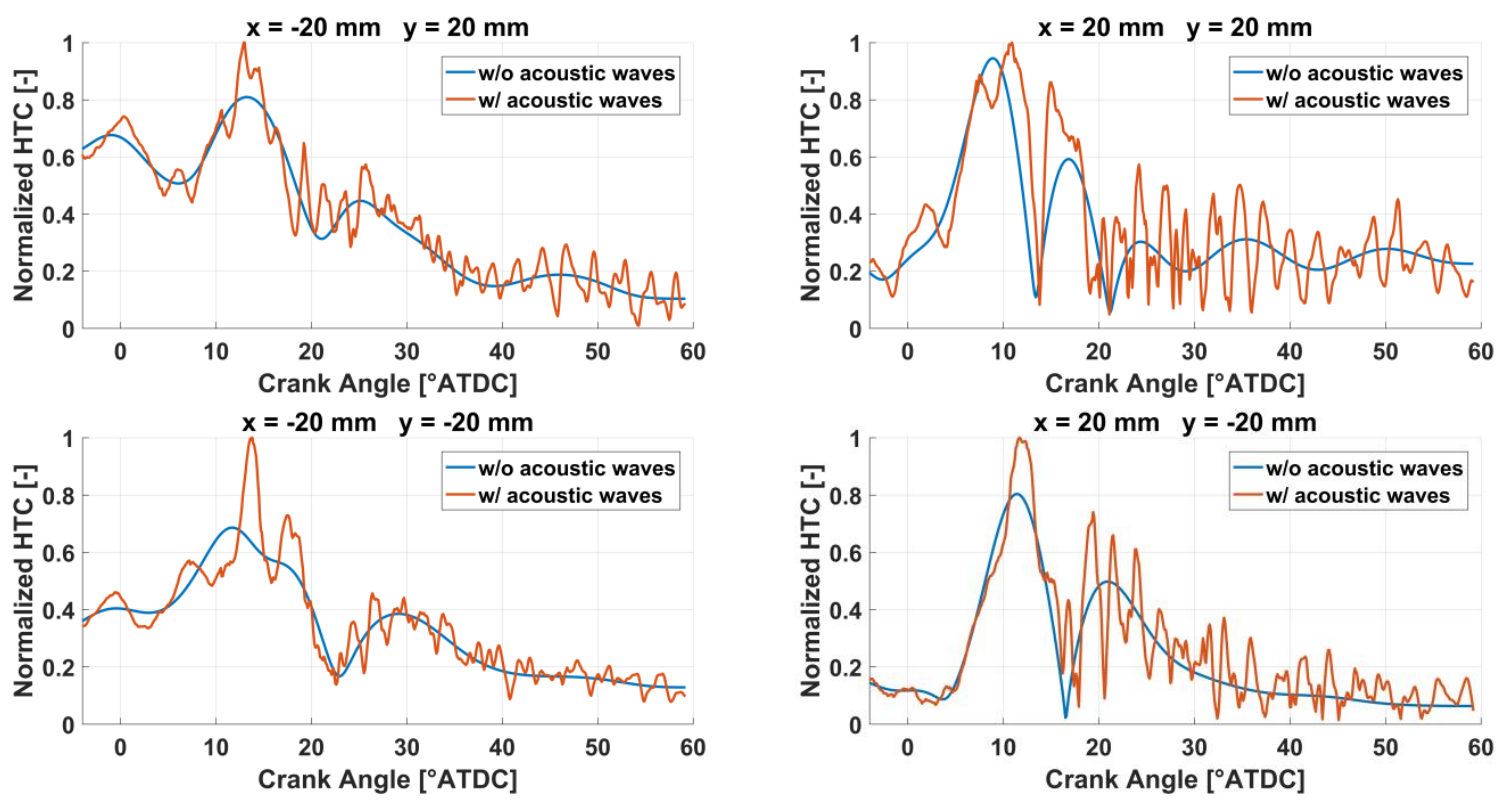

Figure 10. Heat transfer coefficient near piston surface with and without acoustic waves

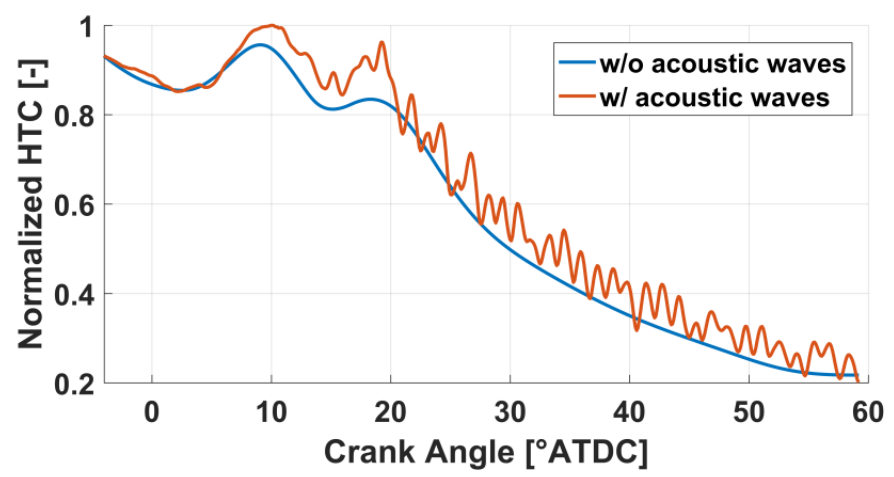

Figure 11. Mean heat transfer coefficient near piston surface with and without acoustic waves

Similar illustrations as Figure 8 and Figure 9 are provided for the heat transfer coefficient (HTC) in Figure 10 and Figure 11. The effect of velocity oscillations are directly observable on the heat transfer coefficient and helps to explain the additional heat transfer in the presence of acoustic waves (pressure oscillations). As previously shown in Figure 1, the conventional model of the heat transfer to the walls underestimates the actual magnitude. As can be observed in Figure 10 and Figure 11, this results primarily from the neglect of the effects of acoustic waves on the heat transfer coefficient. As such, the calculation of the heat transfer coefficient is modified by taking into consideration the additional velocities calculated in Eq. 7-10.

The resulting heat transfer coefficient yields oscillating patterns that stem from the gas velocity oscillations near the piston surface. Experimental studies [28, 5] have also explored this phenomenon (oscillating heat transfer to piston and wall). To the authors' best knowledge, this is among the first study to show simulation results displaying the oscillatory behavior of the heat transfer in knocking conditions. 
The study shows that the calculations of the heat transfer coefficient and the wall heat transfer are more accurate when the effects of the pressure oscillation are properly considered. The study proposes an improved formulation of the heat transfer coefficient through a modified and more accurate representation of the in-cylinder gas velocities.

\title{
Conclusion
}

In this study, a high fidelity LES model incorporating an auto-ignition model is used to analyze the combustion process and the ensuing acoustic waves. It is shown that the incylinder pressure oscillations can be characterized via a simplified modal decomposition. Furthermore, the modal characterization can be utilized for the calculation of in-cylinder velocity oscillations, which play an important role in the heat transfer coefficient.

The study ultimately shows that the calculations of the heat transfer coefficient and the wall heat transfer are more accurate when the effects of the pressure oscillation are properly considered. The increase in heat transfer primarily stems from an increased heat transfer coefficient due to the velocity oscillations of the trapped gas. A series of steps are discussed that relate the pressure oscillations with the velocity oscillations, and ultimately to the wall heat transfer.

The proposed calculation of the heat transfer coefficient (and subsequently the heat transfer to the wall) yields similar trends as those observed experimentally. An oscillating heat transfer is observed with frequencies matching those of the pressure oscillations. Furthermore, an increase of the net heat transfer due to the pressure oscillations is observed, and the contribution from the acoustic waves can be separately quantified.

Future work consists of developing a knock indicator that quantifies the additional heat transfer to the cylinder walls. A new knock quantifying metric, the knock induced heat transfer, is suggested to better reflect the overall conditions in the combustion chamber and better characterize the risks imposed on the engine. Considering that the knock induced heat transfer is a better indicator of the global in-cylinder conditions and the risks of engine damage, the reliance on such a metric allows a better informed knock control strategy.

\section{Nomenclature}

\author{
ATDC - After Top Dead Center \\ AVBP - \\ CA - Crank Angle \\ CHT - Conjugate Heat Transfer \\ CI - Compression Ignited \\ ECFM - Extended Coherent Flame Model \\ HCCI - Homogenous Charge Compression Ignition \\ ISSIM - Imposed Stretch Spark Ignition Model \\ LES - Large Eddy Simulation \\ LLNL - \\ PSD - Power Spectral Density \\ RANS - Reynolds Averaged Navier Stokes \\ SACI - Spark Assisted Compression Ignition
}


SI - Spark Ignited

TKI - Tabulated Kinetics Ignition

TRF - Toluene Reference Fuel 


\section{References}

[1] Z. Wang, H. Liu and R. D. Reitz, "Knocking combustion in spark-ignition engines," Progress in Energy and Combustion Science, vol. 61, pp. 78-112, 2017.

[2] D. K. Srivastava, A. K. Agarwal, A. Datta and M. R. Kumar, Advances in Internal Combustion Engine Research, Singapore: Springer, 2018.

[3] N. Winkler, "Effect of pressure oscillations on in-cylinder heat transfer--through large eddy simulation," International Journal of Engine Research, vol. 16, pp. 705-715, 2015.

[4] E. Gingrich, D. Janecek and J. Ghandhi, "Experimental Investigation of the Impact of InCylinder Pressure Oscillations on Piston Heat Transfer," SAE International Journal of Engines, vol. 9, pp. 1958-1969, 2016.

[5] J.-h. Lu, D. Ezekoye, A. Iiyama, R. Greif and R. F. Sawyer, "Effect of knock on timeresolved engine heat transfer," SAE Technical Paper, 1989.

[6] R. J. Nates and A. D. B. Yates, "Knock damage mechanisms in spark-ignition engines," SAE transactions, pp. 1970-1980, 1994.

[7] M. Kowada, I. Azumagakito, T. Nagai, N. Iwai and R. Hiraoka, "Study of Knocking Damage Indexing Based on Optical Measurement," SAE Technical Paper, 2015.

[8] N. Cavina, N. Rojo, L. Ceschini, E. Balducci, L. Poggio, L. Calogero and R. Cevolani, "Investigation of Knock Damage Mechanisms on a GDI TC Engine," SAE Technical Paper, 2017.

[9] J. Chang, O. Güralp, Z. Filipi, D. N. Assanis, T.-W. Kuo, P. Najt and R. Rask, "New heat transfer correlation for an HCCI engine derived from measurements of instantaneous surface heat flux," SAE transactions, pp. 1576-1593, 2004.

[10] S. Hensel, F. Sarikoc, F. Schumann, H. Kubach and U. Spicher, "Investigations on the heat transfer in HCCI gasoline engines," SAE International Journal of Engines, vol. 2, pp. 1601-1616, 2009.

[11] S. Broekaert, T. De Cuyper, K. Chana, M. De Paepe and S. Verhelst, "Assessment of empirical heat transfer models for a CFR engine operated in HCCI mode," SAE Technical Paper, 2015.

[12] G. Woschni, "A universally applicable equation for the instantaneous heat transfer coefficient in the internal combustion engine," SAE transactions, pp. 3065-3083, 1968.

[13] W. J. D. Annand, "Heat transfer in the cylinders of reciprocating internal combustion 
engines," Proceedings of the Institution of Mechanical Engineers, vol. 177, pp. 973-996, 1963.

[14] G. F. Hohenberg, "Advanced approaches for heat transfer calculations," SAE Technical Paper, 1979.

[15] E. Corti, C. Forte, G. Cazzoli, D. Moro, S. Falfari and V. Ravaglioli, "Comparison of knock indexes based on CFD analysis," Energy Procedia, vol. 101, pp. 917-924, 2016.

[16] A. Robert, K. Truffin, N. Lafrate, S. Jay, O. Colin and C. Angelberger, "Large-eddy simulation analysis of knock in a direct injection spark ignition engine," International Journal of Engine Research, pp. 1-12, 2018.

[17] V. Moureau, G. Lartigue, Y. Sommerer, C. Angelberger, O. Colin and T. Poinsot, "Numerical methods for unsteady compressible multi-component reacting flows on fixed and moving grids," Journal of Computational Physics, vol. 202, pp. 710-736, 2005.

[18] N. Gourdain, L. Gicquel, M. Montagnac, O. Vermorel, M. Gazaix, G. Staffelbach, M. Garcia, J. F. Boussuge and T. Poinsot, "High performance parallel computing of flows in complex geometries: I. methods," Computational Science \& Discovery, vol. 2, p. $015003,2009$.

[19] P. Lax and B. Wendroff, "Systems of conservation laws," Communications on Pure and Applied mathematics, vol. 13, pp. 217-237, 1960.

[20] J. Smagorinsky, "General circulation experiments with the primitive equations: I. The basic experiment," Monthly weather review, vol. 91, pp. 99-164, 1963.

[21] M. García, E. Riber, O. Simonin and T. Poinsot, "Comparison between Euler/Euler and Euler/Lagrange LES approaches for confined bluff-body gas-solid flow prediction," in Proceedings of the international conference on multiphase flow, Leipzig, Germany, 2007.

[22] O. Colin and K. Truffin, "A spark ignition model for large eddy simulation based on an FSD transport equation (ISSIM-LES)," Proceedings of the Combustion Institute, vol. 33, pp. 3097-3104, 2011.

[23] S. Richard, O. Colin, O. Vermorel, A. Benkenida, C. Angelberger and D. Veynante, "Towards large eddy simulation of combustion in spark ignition engines," Proceedings of the Combustion Institute, vol. 31, pp. 3059-3066, 2007.

[24] O. Vermorel, S. Richard, O. Colin, C. Angelberger, A. Benkenida and D. Veynante, "Towards the understanding of cyclic variability in a spark ignited engine using multicycle LES," Combustion and Flame, vol. 156, pp. 1525-1541, 2009. 
[25] O. Colin, A. P. Cruz and S. Jay, "Detailed chemistry-based auto-ignition model including low temperature phenomena applied to 3-D engine calculations," Proceedings of the Combustion Institute, vol. 30, pp. 2649-2656, 2005.

[26] A. Robert, S. Richard, O. Colin, L. Martinez and L. De Francqueville, "LES prediction and analysis of knocking combustion in a spark ignition engine," Proceedings of the Combustion Institute, vol. 35, pp. 2941-2948, 2015.

[27] M. Mehl, W. J. Pitz, C. K. Westbrook and H. J. Curran, "Kinetic modeling of gasoline surrogate components and mixtures under engine conditions," Proceedings of the Combustion Institute, vol. 33, pp. 193-200, 2011.

[28] K. Dejima, O. Nakabeppu, Y. Nakamura and e. al., "Development of metal substrate MEMS sensors for wall heat flux measurement in engines," Transactions of the JSME, vol. 84, no. 858, 2018.

[29] S. M. Candel, Analytical studies of some acoustic problems of jet engines, $\mathrm{PhD}$ Thesis : California Institute of Technology, 1972.

[30] A. J. Shahlari and J. Ghandhi, "Pressure-Based Knock Measurement Issues," SAE Technical Paper, 2017.

[31] C. S. Draper, "The physical effects of detonation in a closed cylindrical chamber," 1935.

[32] C. S. Draper, "Pressure waves accompanying detonation in the internal combustion engine," Journal of the Aeronautical Sciences, vol. 5, pp. 219-226, 1938.

[33] A. Vressner, A. Lundin, M. Christensen, P. Tunestål and B. Johansson, "Pressure oscillations during rapid HCCI combustion," SAE transactions, pp. 2469-2478, 2003.

[34] C. Guardiola, B. Pla, D. Blanco-Rodriguez and P. Bares, "Cycle by cycle trapped mass estimation for diagnosis and control," SAE International Journal of Engines, vol. 7, pp. 1523-1531, 2014.

[35] M. M. Andreae, W. K. Cheng, T. Kenney and J. Yang, "On HCCI engine knock," SAE Transactions, pp. 355-363, 2007.

[36] S. M. Dues, J. M. Adams and G. A. Shinkle, "Combustion knock sensing: sensor selection and application issues," SAE transactions, pp. 1192-1202, 1990.

[37] M. Sjoberg and X. He, "Combined effects of intake flow and spark-plug location on flame development, combustion stability and end-gas autoignition for lean spark-ignition engine operation using E30 fuel," International Journal of Engine Research, vol. 19, pp. 86-95, 2018. 
[38] A. Poubeau, A. Vauvy, F. Duffour, J.-M. Zaccardi, G. de Paola and M. Abramczuk, "Modeling investigation of thermal insulation approaches for low heat rejection Diesel engines using a conjugate heat transfer model," International Journal of Engine Research, vol. 20, pp. 92-104, 2019.

[39] C. Netzer, L. Seidel, F. Ravet and F. Mauss, "Assessment of the validity of RANS knock prediction using the resonance theory," International Journal of Engine Research, pp. 112, 2019. 